TITLE:

\title{
Entropy Governed by the Absorbing State of Directed Percolation
}

$\operatorname{AUTHOR}(S)$ :

Harada, Kenji; Kawashima, Naoki

CITATION:

Harada, Kenji ... [et al]. Entropy Governed by the Absorbing State of

Directed Percolation. Physical Review Letters 2019, 123(9): 090601.

ISSUE DATE:

2019-08-30

URL:

http://hdl.handle.net/2433/243891

RIGHT: 


\title{
Entropy Governed by the Absorbing State of Directed Percolation
}

\author{
Kenji Harada,* \\ Graduate School of Informatics, Kyoto University, Kyoto 606-8501, Japan \\ Naoki Kawashima \\ Institute for Solid State Physics, University of Tokyo, Kashiwa, Chiba 277-8581, Japan
}

(Received 8 April 2019; revised manuscript received 13 June 2019; published 27 August 2019)

\begin{abstract}
We investigate the informational aspect of $(1+1)$-dimensional directed percolation, a canonical model of a nonequilibrium continuous transition to a phase dominated by a single special state called the "absorbing" state. Using a tensor network scheme, we numerically calculate the time evolution of state probability distribution of directed percolation. We find a universal relaxation of Rényi entropy at the absorbing phase transition point as well as a new singularity in the active phase, slightly but distinctly away from the absorbing transition point. At the new singular point, the second-order Rényi entropy has a clear cusp. There we also detect a singular behavior of "entanglement entropy," defined by regarding the probability distribution as a wave function. The entanglement entropy vanishes below the singular point and stays finite above. We confirm that the absorbing state, though its occurrence is exponentially rare in the active phase, is responsible for these phenomena. This interpretation provides us with a unified understanding of time evolution of the Rényi entropy at the critical point as well as in the active phase.
\end{abstract}

DOI: 10.1103/PhysRevLett.123.090601

Introduction.-We can define the phase of a macroscopic system out of equilibrium based on physical order parameters of a nonequilibrium steady-state distribution. The phase transition is confirmed theoretically and experimentally in many studies [1-9]. However, we have little understanding of the informational aspect of a macroscopic nonequilibrium system. Recently, Wood et al. [10] analytically calculated the statistical Rényi entropy of the asymmetric exclusion process $[1,2,8]$. The phase boundary defined by the behavior of the Rényi entropy agrees with the conventional phase boundary of the asymmetric exclusion process for three nonequilibrium phases. It shows the potential power of informational quantities. However, computation of the entropy is technically difficult; no method for directly measuring entropy is known for the Monte Carlo simulation of a nonequilibrium system. Therefore, the knowledge about the informational aspect of a nonequilibrium system is limited than that of an equilibrium system [11-13].

In this study, overcoming the technical difficulty by the tensor network method, we will focus on the informational aspect of a $(1+1)$-dimensional directed percolation (DP) through the Rényi entropy and the entanglement entropy. While the Rényi entropy is discussed frequently in conventional statistics, the introduction of the entanglement entropy to the analysis of the classical model is somehow unconventional. Its utility in the context of the classical models was recently discussed $[14,15]$. If we regard a percolating direction of objects as a time direction, the $(d+1)$-dimensional DP corresponds to the $d$-dimensional reaction-diffusion process. There are generally two phases in a reaction-diffusion process, the active phase with finite density of objects and the inactive phase with zero density in the long-time limit. As shown in many numerical studies for DP (see a review [9]), the transition between the active phase and the inactive phase is critical, and the concept of the universality is also extended as the DP universality class. Because of the extreme simplicity, the universality class is expected to be ubiquitous [4-7,16-18]. In the following, after we first briefly introduce the model of DP and the numerical method for the Rényi entropy and the entanglement entropy, we will report the results of both entropies as well as their interpretations.

Directed percolation.-A $(1+1)$-dimensional DP is defined on a square lattice rotated by $45^{\circ}$. A site can be either active or inactive. An active state can percolate to the nearest-neighbor sites with a probability $p$. However, the direction of percolation is limited. If it is downward, there are two nearest-neighbor sites from which a site may be percolated. We can regard the row of sites as a onedimensional system at a time. Then, the DP describes a reaction-diffusion process in a one-dimensional system. This DP is called a bond DP, and it is a special case of Domany-Kinzel (DK) automaton [3]. In the DK automaton, a probability of an active state depends on the number of active states $n$ in the nearest-neighbor sites at the previous time as $P[n]$. If there is no active nearest neighbor just before the current time, the probability of having an active state is zero, i.e., $P[0]=0$. The bond DP is defined as $P[1]=p, P[2]=p(2-p)$. There is a special state in a DK 
automaton in which there is no active site. It is called the absorbing state because a system cannot escape from it. If $P[1]$ and $P[2]$ are small, the state of the system eventually arrives at the absorbing state after a long time. Then, the evolution of the automaton is stopped. If $P[1]$ and $P[2]$ are large enough, the steady-state distribution with other states can exist in the thermodynamic limit. Thus, there are an active phase and an inactive phase in a DK automaton, and the nonequilibrium phase transition between them is critical. It is called an absorbing phase transition. The behavior at the critical point of DK automatons is universal. It is called the DP universality class.

Numerical method for Rényi and entanglement entropies.-A state probability distribution $P(S)$ can be mapped to a normalized "wave function" [19] of which amplitude is proportional to a state probability distribution as $|\psi(t)\rangle=\exp \left[H_{2}(t) / 2\right] \sum_{S} P(S)|S\rangle$. Here, $H_{2}$ is the second-order Rényi entropy [20]. It is a generalization of the Shannon entropy and is defined as $H_{q}=$ $-(q-1)^{-1} \log \sum_{S}[P(S)]^{q}$, where $q$ is called the order. The Shannon entropy is recovered when $q \rightarrow 1$.

Recently, various new techniques have been developed in the calculation of a wave function. The most promising approach is the use of a tensor network representation which is a composite tensor defined by tensor contractions. Here, we use a one-dimensional tensor network, i.e., matrix product states (MPS), to represent a wave function of a onedimensional system. Since the transfer matrix in a master equation is an operator to the wave function which is written as a tensor network [21], we can calculate the time step evolution of a wave function as a tensor contraction between them [22-24]. Holding a canonical form of MPS [25], we can efficiently calculate an approximated MPS at the next time step by the time-evolving block decimation method $[21,26,27]$. We can control the precision of MPS representation by a bond dimension of the virtual index between two tensors in MPS [21]. Since the second-order Rényi entropy appears in a normalization factor of a wave function, we can efficiently calculate it from a tensor contraction of two MPSs.

Through the above mapping between a distribution and a wave function, we can formally introduce the concept of entanglement of a quantum state into a state probability distribution. The entanglement entropy of a quantum state is the Shannon entropy of a quantum subsystem as $H_{E}(A)=-\operatorname{Tr} \rho_{A} \log \rho_{A}$, where $\rho_{A}$ is a reduced density operator of a subsystem $A$. The entanglement entropy quantifies a quantum correlation between a subsystem $A$ and its complement $\bar{A}$. In general, we can define the Schmidt decomposition of a quantum state as $|\psi\rangle=$ $\sum_{k} e^{-(1 / 2) \xi_{k}}\left|\psi_{A}^{k}\right\rangle \otimes\left|\psi_{\bar{A}}^{k}\right\rangle$, where $\left|\psi_{A}^{k}\right\rangle$ and $\left|\psi_{\bar{A}}^{k}\right\rangle$ are orthogonal states in subsystem $A$ and its complement $\bar{A}$, respectively. Then, the entanglement entropy is rewritten as $H_{E}(A)=\sum_{k} \xi_{k} \exp \left(-\xi_{k}\right)$ and $\left\{\xi_{k}\right\}$ is called an entanglement spectrum. Since the entanglement spectrum is a key component in a canonical form of MPS, we can efficiently calculate it from the canonical form of MPS.

Critical relaxation of Rényi entropy.-The density of active sites is an order parameter of DP. If we start from a homogeneously full active initial state, the density of active sites shows a power-law decay at the critical point. For example, critical points of the bond DP and the site DP are at $p_{c}=0.644700185(5)$ and $0.70548515(20)$, respectively [28]. The decay exponent is universal in the DP universality class. Figure 1 shows the critical relaxation of the density and the Rényi entropy per site at the critical points of the bond DP and the site DP of 8192 sites with open boundary condition. The tensor network with the bond dimension 250 was used for the calculation. Here, to reduce the boundary effect, the density is measured at the central site, whereas the Rényi entropy is defined on the central half of the system. The site DP is a special case in DK automaton as $P[0]=0, P[1]=P[2]=p$, and it also belongs to the $\mathrm{DP}$ universality class. Thus, the decay exponents for both DPs should be equal. Our estimates of the decay exponent based on Fig. $1, \delta_{\text {bond }}=0.1593(1)$ for the bond DP and $\delta_{\text {site }}=$ 0.158 (1) for the site DP. They are consistent with a previously estimated value $\delta=0.159464$ [29] within the error. Thus, we confirm the reliability of MPS calculation for criticality. The Rényi entropy per site is also an order parameter of the absorbing phase transition because it takes a finite value only in the active phase. As shown in Fig. 1, Rényi entropy at the critical point also shows a power-law decay. Since the decay exponents in Fig. 1 are 0.632(5) and 0.625 (7) for bond DP and site DP, respectively, we confirm the universal relaxation in the Rényi entropy. As we see below, this universal relaxation can be expected as

$$
h_{2}(t)=H_{2}(t) / N \sim \xi_{\perp}(t)^{-1} \sim t^{-1 / z},
$$

where $\xi_{\perp}$ is the spatial correlation length. Indeed, the value of decay exponent in Fig. 1 is consistent with the reciprocal of the dynamical critical exponent, $1 / z=0.632613(6)$ [29].

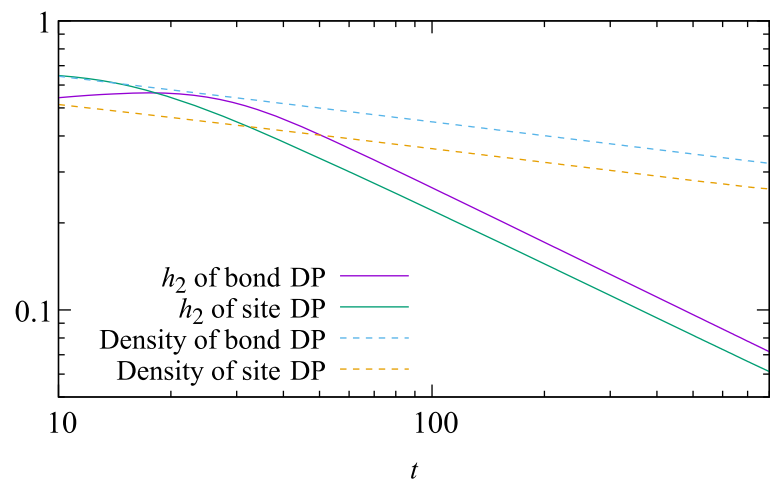

FIG. 1. Time evolution of the second-order Rényi entropy per site $h_{2}$ (solid) and the density of active sites (dashed) at critical points of bond DP and site DP. 


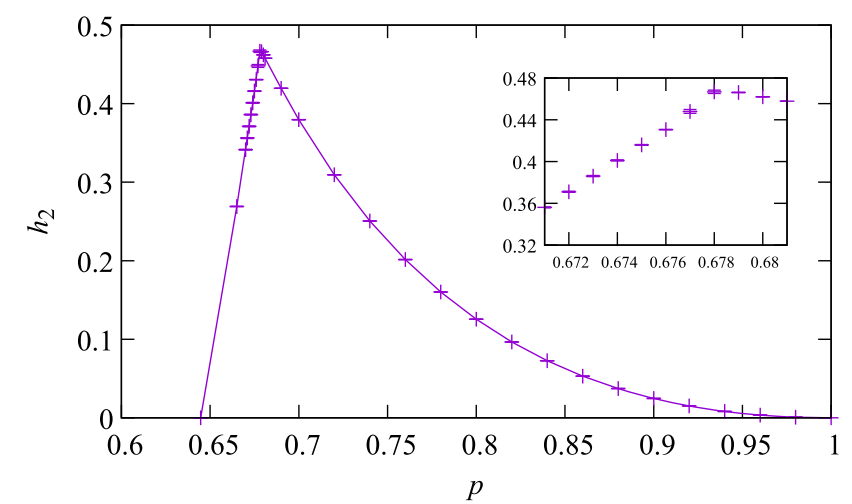

FIG. 2. The second-order Rényi entropy per site $h_{2}$ of the steady-state distribution of the bond DP in the thermodynamic limit. There is a clear cusp slightly above the bond-DP threshold, $p_{c}=0.644700185(5)$ [28]. The inset is the enlarged view near $p_{2}^{*}=0.6785(5)$.

Rényi entropy and entanglement entropy in active phase.-If the initial probability distribution consists of a fully active state, the Rényi entropy per site starts from zero. In the early time region, it rapidly grows. After that, it quickly converges to a finite value in the active phase. Figure 2 shows the Rényi entropy per site extrapolated to the thermodynamic limit by a $1 / N$ quadratic fitting for a system size $N \leq 4096$ [21]. As shown in the inset, there is a cusp at $p_{2}^{*}=0.6785(5)$ in the active phase.

We calculate the time evolution of entanglement entropy between the left and the right halves of the system from the fully active initial state. Figure 3 shows entanglement entropy from the system size 128 to 8192 . The bond dimension in MPS is 50 [30]. Solid and dashed lines represent entanglement entropy of $p=0.678$ and 0.679 , respectively. The entanglement entropy has a peak for both cases. The peak time of $p=0.678$ almost converges for

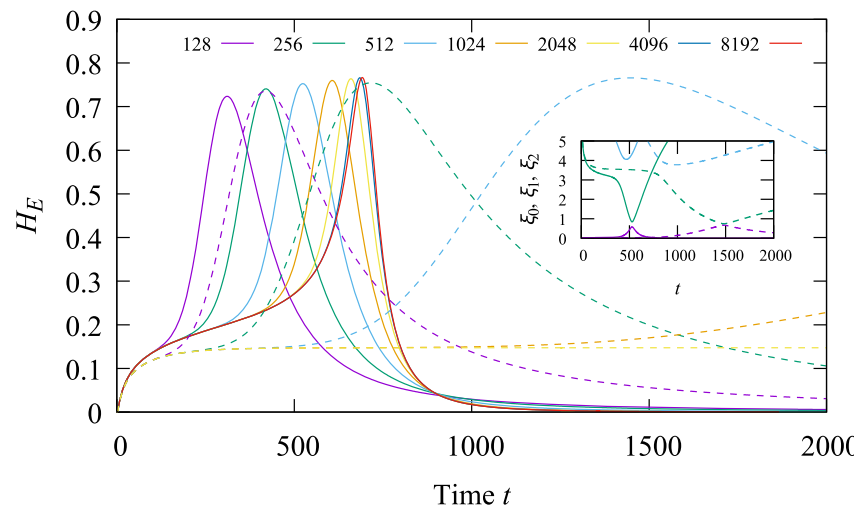

FIG. 3. Time evolution of entanglement entropy $H_{E}$ between the left and the right halves of the system from system size 128 to 8192. Solid and dashed lines represent $H_{E}$ at $p=0.678$ and 0.679 , respectively. Solid and dashed lines in the inset represent singular values $\xi_{0}, \xi_{1}$, and $\xi_{2}$ at $p=0.678$ and 0.679 for 512 sites, respectively. more than 4096 sites. However, the peak time of $p=0.679$ diverges with the system size. Solid and dashed lines in the inset represent the levels in the entanglement spectrum from $\xi_{0}$ to $\xi_{2}$ for $p=0.678$ and 0.679 with 512 sites, respectively. The crossover time at which two levels $\xi_{0}$ and $\xi_{1}$ cross agrees with the peak time of entanglement entropy. Figure 4 shows the crossover time $\tau_{0}$ from $p=0.676$ to 0.681 for less than 8192 sites. When we increase the system size, $\tau_{0}$ diverges for $p \geq 0.6786$. However, $\tau_{0}$ converges or its increase becomes weaker for $p \leq 0.6785$. In the thermodynamic limit, the entanglement entropy has a finite peak time for $p<p_{E}^{*}=0.67855(5)$, but no peak for $p>p_{E}^{*}$. The asymptotic behavior of entanglement entropy also changes at $p_{E}^{*}$. In a long time region, the entanglement entropy eventually decreases and converges to zero for $p<p_{E}^{*}$. On the other hand, it converges to a finite value for $p>p_{E}^{*}$. As shown in the inset, the asymptotic behavior of a gap $\Delta_{0}$ between $\xi_{0}\left(\tau_{0}\right)$ and $\xi_{1}\left(\tau_{0}\right)$ also changes at $p_{E}^{*}$. The gap is open for $p<p_{E}^{*}$, but it is closed for $p>p_{E}^{*}$. The position of a singularity of entanglement entropy $p_{E}^{*}$ is equal to the position of a singularity of the second-order Rényi entropy $p_{2}^{*}$ within the error. As we see below, these singularities can be explained by the effect of the extremely rare absorbing state.

Effect of absorbing state.-The probability of the absorbing state monotonically increases, because the system cannot escape from it. Therefore, the absorbing state is the only steady state for any finite system. To define a nontrivial steady state, we must consider an infinite system. We then consider a marginal distribution for a subsystem that consists of sequential $N$ sites. In the long time limit, it safely converges to a steady-marginal distribution $P_{N}(S)$. From our calculation, we observe that this steady-state marginal distribution consists of two components, the delta function at the zero-density state and the continuous component around some nonzero density. Namely,

$$
P_{N}(S)=\delta_{S=0} P_{N}(0)+\delta_{S \neq 0}\left[1-P_{N}(0)\right] Q_{N}(S) .
$$

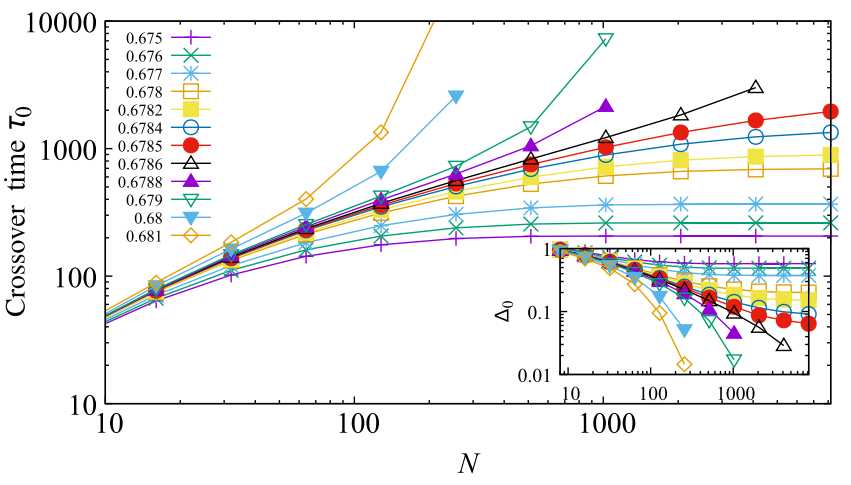

FIG. 4. Crossover time $\tau_{0}$ between two levels $\xi_{0}$ and $\xi_{1}$ from $p=0.676$ to 0.681 . $N$ denotes a system size. The inset shows a gap $\Delta_{0}$ between $\xi_{0}\left(\tau_{0}\right)$ and $\xi_{1}\left(\tau_{0}\right)$. 
The delta-function component can be interpreted as a "quasiabsorbing" state, which is a consequence of the fact that the zero-density state plays a special role even in the marginal distribution because of the absence of the nucleation; i.e., active sites occur only by the influence from the outside of the boundary. Here, since the occurrence of the absorbing state is exponentially rare in the active phase, we assume an asymptotic form of the probability of quasiabsorbing state as

$$
P_{N}(0) \sim \exp \left[-\sigma_{0}\left(N / \xi_{\perp}\right)\right],
$$

where $\exp \left(-\sigma_{0}\right)$ is the probability of having the zerodensity state in a correlated region where $\xi_{\perp}$ is the region's size. In other words, $\sigma_{0}$ is the renormalized chemical potential (of holes).

In the active phase, the Rényi entropy per site of a continuous-part $Q_{N}$ converges to $h_{q}^{\prime} \equiv$ $-(q-1)^{-1} \log \sum_{S}\left[Q_{N}(S)^{q}\right] / N$. Using (3), Rényi entropy per site for a steady-marginal distribution in the large $N$ limit is written as

$$
h_{q}=\min \left[\{q /(q-1)\}\left(\sigma_{0} / \xi_{\perp}\right), h_{q}^{\prime}\right] .
$$

The singularity at $p_{q}^{*}$ can be understood as the crossover from the first-term dominant region to the second term dominant one, so that $\{q /(q-1)\}\left\{\sigma_{0}\left(p_{q}^{*}\right) / \xi_{\perp}\left(p_{q}^{*}\right)\right\}=$ $h_{q}^{\prime}\left(p_{q}^{*}\right)[31]$.

The entanglement entropy is the Shannon entropy for the singular value part of the amplitude matrix $\mathbb{P}$ as $\mathbb{P}_{S_{L}, S_{R}}=$ $\left\langle S_{L}, S_{R} \mid \psi\right\rangle$, where $S_{L}$ and $S_{R}$ denote states of left and right half systems. Based on (2), $\mathbb{P}=\mathbb{P}^{0}+\mathbb{Q}$, where $\mathbb{P}^{0} S_{L}, S_{R}=$ $\exp \left(N h_{2} / 2\right) P_{N}(0) \delta_{S_{L}=S_{R}=0}$. From (4), the Frobenius norm [32] $\left|\mathbb{P}^{0}\right| \gg|\mathbb{Q}|$ for $p<p_{2}^{*}$, and vice versa in the large $N$ limit. The quasiabsorbing state is a direct product state. Therefore, the entanglement entropy is zero for $p<p_{2}^{*}$ and may be finite for $p>p_{2}^{*}$. This result is consistent with the common singular point for the entanglement entropy and the second-order Rényi entropy in the bond DP.

So far, we have been discussing singularity of the entropies due to the crossover from the "absorbing-state dominating" regime to the "continuous-part dominating" regime. While the transitions discussed above are fictitious, the "real" critical phenomena can also be understood by this crossover. To see this, we extend (3) to the probability $P_{N}(0, t)$ allowing the time dependence of the correlation length as

$$
P_{N}(0, t) \sim \exp \left[-\sigma_{0} N / \xi_{\perp}(t)\right] .
$$

As shown in Fig. 5, the absorbing-state entropy $-2 \log P_{N}(0, t) / N$, equal to the first term of (4), is a decreasing function of time, because $\xi_{\perp}(t)$ monotonically increases. For $p=0.68>p_{2}^{*}=0.6785(5)$, it never completely catches up with $h_{2}(t)$. When $p_{c}<p=0.67<p_{2}^{*}$,

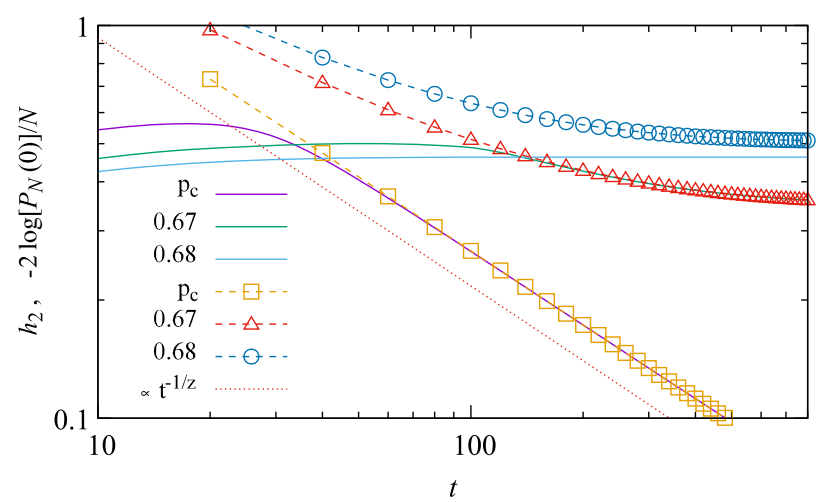

FIG. 5. Comparison between the second-order Rényi entropy per site $h_{2}$ (solid lines) and the absorbing-state entropy $-2 \log \left[P_{N}(0)\right] / N$ (symbols).

it catches up with it at a certain point of time and once it does it dominates the Renyi entropy; $h_{2}$ and the absorbingstate entropy agree with each other afterward. These are the behaviors that are naturally expected from (4) and (5). However, this behavior remains essentially the same even at the critical point $p=p_{c}$, with the only difference being that the asymptotic curve is power-law decay in (1). As shown in Fig. 5, the agreement between the slope of the asymptotic behavior and $z$ confirms the validity of the interpretation with the simple assumption (5).

Conclusion.-We focus on the informational aspect of the $(1+1)$-dimensional DP through a statistical Rényi entropy and an entanglement entropy. We numerically find a universal relaxation with a slope $1 / z$ at the critical point $p_{c}$, and a cusp at $p_{2}^{*}=0.6785(5)$ in the active phase of the bond DP for the second-order Rényi entropy. As we increase $p$ passing $p_{2}^{*}$, the dynamical behavior of the entanglement entropy also changes. The entanglement entropy asymptotically goes to zero below $p_{2}^{*}$ and converges to a finite value above $p_{2}^{*}$. Based on the assumption (5) for the asymptotic form of the probability of quasiabsorbing state, we can understand all of their behavior as the domination of the absorbing state of which the occurrence is exponentially rare in the active phase. Since the existence of the absorbing state is the unique character of DP, the universal relaxation and the cusp in the Rényi entropy are hallmarks of DP [21]. Our scenario can be extended to the higher-dimensional DP and the DP-like models.

In this study, we show an application of a tensor network scheme to DP as a canonical model of a nonequilibrium continuous transition. The tensor network scheme gives us detailed information of the state probability distribution as the Rényi entropy. We hope for the further use of tensor networks to understand the characterization of nonequilibrium systems from the informational point of view.

K. H. appreciates fruitful comments from T. Okubo and K. Tamai, and K. H. acknowledges many discussions with R. Sato and K. Nataochi about the numerical schemes in the 
early stage of this study. This work was supported by JSPS KAKENHI Grant No. 17K05576, and by MEXT as Exploratory Challenge on Post-K computer (Frontiers of Basic Science: Challenging the Limits). The computation in this work has been done using the facilities of the Supercomputer Center, the Institute for Solid State Physics, the University of Tokyo and the facilities of the Supercomputer Center at Kyoto University.

*Corresponding author. harada@i.kyoto-u.ac.jp

[1] B. Derrida, M. R. Evans, V. Hakim, and V. Pasquier, Exact solution of a 1D asymmetric exclusion model using a matrix formulation, J. Phys. A 26, 1493 (1993).

[2] T. Sasamoto, One-dimensional partially asymmetric simple exclusion process with open boundaries: Orthogonal polynomials approach, J. Phys. A 32, 7109 (1999).

[3] E. Domany and W. Kinzel, Equivalence of Cellular Automata to Ising Models and Directed Percolation, Phys. Rev. Lett. 53, 311 (1984).

[4] K. A. Takeuchi, M. Kuroda, H. Chaté, and M. Sano, Directed Percolation Criticality in Turbulent Liquid Crystals, Phys. Rev. Lett. 99, 234503 (2007).

[5] K. A. Takeuchi, M. Kuroda, H. Chaté, and M. Sano, Experimental realization of directed percolation criticality in turbulent liquid crystals, Phys. Rev. E 80, 051116 (2009).

[6] M. Sipos and N. Goldenfeld, Directed percolation describes lifetime and growth of turbulent puffs and slugs, Phys. Rev. E 84, 035304(R) (2011).

[7] M. Sano and K. Tamai, A universal transition to turbulence in channel flow, Nat. Phys. 12, 249 (2016).

[8] B. Derrida, Non-equilibrium steady states: Fluctuations and large deviations of the density and of the current, J. Stat. Mech. (2007) P07023.

[9] M. Henkel, H. Hinrichsen, and S. Lübeck, Non-Equilibrium Phase Transitions (Springer, New York, 2008), Vol. 1.

[10] A. J. Wood, R. A. Blythe, and M. R. Evans, Rényi entropy of the totally asymmetric exclusion process, J. Phys. A 50, 475005 (2017).

[11] L. Barnett, J. T. Lizier, M. Harré, A. K. Seth, and T. Bossomaier, Information Flow in a Kinetic Ising Model Peaks in the Disordered Phase, Phys. Rev. Lett. 111, 177203 (2013).

[12] J. Iaconis, S. Inglis, A. B. Kallin, and R. G. Melko, Detecting classical phase transitions with Renyi mutual information, Phys. Rev. B 87, 195134 (2013).

[13] J.-M. Stéphan, S. Inglis, P. Fendley, and R. G. Melko, Geometric Mutual Information at Classical Critical Points, Phys. Rev. Lett. 112, 127204 (2014).

[14] M. C. Bañuls and J. P. Garrahan, Using matrix product states to study the dynamical large deviations of kinetically constrained models, arXiv:1903.01570.

[15] P. Helms, U. Ray, and G.K-L. Chan, Dynamical phase behavior of the single- and multi-lane asymmetric simple exclusion process via matrix product states, Phys. Rev. E 100, 022101 (2019).
[16] F. Vazquez, J. A. Bonachela, C. López, and M. A. Muñoz, Temporal Griffiths Phases, Phys. Rev. Lett. 106, 235702 (2011).

[17] C. I. N. Sampaio Filho, J. S. Andrade, Jr., H. J. Herrmann, and A. A. Moreira, Elastic Backbone Defines a New Transition in the Percolation Model, Phys. Rev. Lett. 120, 175701 (2018).

[18] Y. Deng and R. M. Ziff, The elastic and directed percolation backbone, arXiv:1805.08201.

[19] M. Doi, Second quantization representation for classical many-particle system, J. Phys. A 9, 1465 (1976).

[20] A. Rényi, On measures of entropy and information, in Proceedings of the Fourth Berkeley Symposium on Mathematical Statistics and Probability, edited by J. Neyman (University of California Press, Berkeley, 1961), Vol. 1, pp. 547-561.

[21] See Supplemental Material at http://link.aps.org/ supplemental/10.1103/PhysRevLett.123.090601 for (i) the exact tensor network representation of a transfer matrix of the Domany-Kinzel automaton; (ii) the canonical form of a matrix product state; (iii) the time evolution of a density in the bond DP; (iv) the Rényi entropy of the steady-state distribution of the bond DP in the thermodynamic limit; and (v) the Rényi entropy of the steady-state distribution of the site DP in the thermodynamic limit.

[22] T. H. Johnson, S. R. Clark, and D. Jaksch, Dynamical simulations of classical stochastic systems using matrix product states, Phys. Rev. E 82, 036702 (2010).

[23] T. H. Johnson, T. J. Elliott, S. R. Clark, and D. Jaksch, Capturing Exponential Variance Using Polynomial Resources: Applying Tensor Networks to Nonequilibrium Stochastic Processes, Phys. Rev. Lett. 114, 090602 (2015).

[24] Y. Hotta, Tensor-network algorithm for nonequilibrium relaxation in the thermodynamic limit, Phys. Rev. E 93, 062136 (2016).

[25] Y. Y. Shi, L. M. Duan, and G. Vidal, Classical simulation of quantum many-body systems with a tree tensor network, Phys. Rev. A 74, 022320 (2006).

[26] G. Vidal, Efficient Classical Simulation of Slightly Entangled Quantum Computations, Phys. Rev. Lett. 91, 147902 (2003).

[27] G. Vidal, Efficient Simulation of One-Dimensional Quantum Many-Body Systems, Phys. Rev. Lett. 93, 040502 (2004).

[28] I. Jensen, Low-density series expansions for directed percolation: III. Some two-dimensional lattices, J. Phys. A 37, 6899 (2004).

[29] I. Jensen, Low-density series expansions for directed percolation: I. A new efficient algorithm with applications to the square lattice, J. Phys. A 32, 5233. (1999).

[30] Results in Fig. 3 do not change when the bond dimension is larger than 50.

[31] Based on (4), we can expect the singular behavior of the Rényi entropy near $p_{c}$ with the exponent $\nu_{\perp}$. The value of the exponent estimated based on all points of Fig. 2 below $p_{2}^{*}$ is 1.093(5). Our estimation of the exponent is consistent with the value of $\nu_{\perp}=1.096854(4)$ [29].

[32] The Frobenius norm of matrix $\mathbb{A}$ is $\sqrt{\operatorname{Tr} \mathbb{A}^{\dagger} \mathbb{A}}$. It is the square root of the summation of squared singular values. 